This is the post peer-review accepted manuscript of:

G. Tagliavini, S. Mach, D. Rossi, A. Marongiu and L. Benini, "A transprecision floating-point platform for ultra-low power computing," 2018 Design, Automation \& Test in Europe Conference \& Exhibition (DATE), Dresden, 2018, pp. 1051-1056. doi: 10.23919/DATE.2018.8342167

The published version is available online at: https://doi.org/10.23919/DATE.2018.8342167

C 2018 IEEE. Personal use of this material is permitted. Permission from IEEE must be obtained for all other uses, in any current or future media, including reprinting/republishing this material for advertising or promotional purposes, creating new collective works, for resale or redistribution to servers or lists, or reuse of any copyrighted component of this work in other works 


\title{
A Transprecision Floating-Point Platform for Ultra-Low Power Computing
}

\author{
Giuseppe Tagliavini*, Stefan Mach $^{\dagger}$, Davide Rossi*, Andrea Marongiu ${ }^{\ddagger}$, and Luca Benini*† \\ * DEI, University of Bologna, Italy / Email: \{giuseppe.tagliavini, davide.rossi, luca.benini\}@ unibo.it \\ $\dagger$ IIS, ETH Zurich, Switzerland / Email: \{smach, luca.benini\}@iis.ee.ethz.ch \\ $\ddagger$ DISI, University of Bologna, Italy / Email: \{a.marongiu\}@unibo.it
}

\begin{abstract}
In modern low-power embedded platforms, the execution of floating-point (FP) operations emerges as a major contributor to the energy consumption of compute-intensive applications with large dynamic range. Experimental evidence shows that $50 \%$ of the energy consumed by a core and its data memory is related to $F P$ computations. The adoption of FP formats requiring a lower number of bits is an interesting opportunity to reduce energy consumption, since it allows to simplify the arithmetic circuitry and to reduce the memory bandwidth required to transfer data between memory and registers by enabling vectorization. From a theoretical point of view, the adoption of multiple FP types perfectly fits with the principle of transprecision computing, allowing fine-grained control of approximation while meeting specified constraints on the precision of final results. In this paper we propose an extended FP type system with complete hardware support to enable transprecision computing on low-power embedded processors, including two standard formats (binary32 and binary16) and two new formats (binary8 and binary16alt). First, we introduce a software library that enables exploration of FP types by tuning both precision and dynamic range of program variables. Then, we present a methodology to integrate our library with an external tool for precision tuning, and experimental results that highlight the clear benefits of introducing the new formats. Finally, we present the design of a transprecision FP unit capable of handling 8-bit and 16-bit operations in addition to standard 32bit operations. Experimental results on FP-intensive benchmarks show that up to $90 \%$ of FP operations can be safely scaled down to 8-bit or 16-bit formats. Thanks to precision tuning and vectorization, execution time is decreased by $12 \%$ and memory accesses are reduced by $27 \%$ on average, leading to a reduction of energy consumption up to $30 \%$.
\end{abstract}

\section{INTRODUCTION}

Nowadays most embedded applications involving numerical computations with large dynamic range are performed using binary64 (double-precision) or binary32 (single-precision) floating-point (FP) formats, described by the IEEE 754 standard [18]. In these applications, the execution of FP operations emerges as a major contributor to the energy consumption. To provide experimental evidence of this insight, we have executed a set of FP-intensive applications on PULPino [7], an open-source ULP microcontroller. Results show that $30 \%$ of the energy consumption of the core is actually due to FP operations. Moreover, an additional $20 \%$ is spent in moving FP operands from data memory to registers and vice versa.

To provide a compromise between energy cost and dynamic range, IEEE 754 introduces a 16-bit format referred to as binaryl6 (half-precision). The introduction of binaryl6 represents a first step to increase the energy efficiency of FP computations, but software development flows for ULP systems still lack a methodology to evaluate the effect of reduced-precision FP variables on application requirements. In practice, programmers often use the maximum precision provided by target platforms, following the most conservative principle: guaranteeing numerical precision of each elementary step also guarantees the precision of final results.

In recent years, significant advances in the field of approximate computing have been made, aimed at relaxing this precise-computing abstraction [2] [12] [10] [4]. The most promising research trends are stepping beyond of the concept of approximation itself, towards a novel paradigm - transprecision computing - in which rather than tolerating errors implied by imprecise HW or SW computations, systems are explicitly designed to deliver just the required precision for intermediate computations. In other words, the specified constraints on the precision of final results are always met (i.e., results are not generically "approximated", they match a required precision), but intermediate operations can be deployed to custom, lowerprecision compute units to save energy.

In this paper we propose an extended FP type system with complete hardware support to enable transprecision computing on ULP embedded platforms. We propose the introduction of two additional formats, namely binary8 and binaryl6alt. Specifically, binary8 is a 8-bit format with low precision (3-bit mantissa), while binaryl6alt is a 16-bit format complementary to the IEEE one and featuring a higher dynamic range (8bit exponent). To assess the benefits of this extended FP type system, we performed a precision analysis supported by additional considerations on the hardware design. As a first step we designed a $\mathrm{C}++$ library to explore the effects on application behavior when varying dynamic range and precision of program variables. Then we adapted a set of applications representative of FP-intensive computations in the embedded domain, adopting emulated FP types and providing an interface with an external tool for precision analysis [10].

Our results shows that the introduction of binary 8 guarantees the best trade-off between precision and dynamic range for applications that match minimum precision requirements. Moreover, the introduction of binaryl6alt extends (up to 50\%) the number of variables that can be safety scaled from a 32-bit representation to a 16-bit one. To provide support at hardware level, we designed a dedicated transprecision FP unit. Our design also enables vectorial operations on smaller-than-32-bit formats, further increasing energy efficiency and performance of the core and reducing pressure on data memory.

To summarize, the main contributions of this work are (i) the introduction of a software library that enables explorations of FP types, (ii) a methodology to integrate our library with external tools for precision tuning, and (iii) an energy-efficient hardware design supporting multiple FP types. Experimental results show that up to $90 \%$ of FP operations can be safely scaled down to 8-bit or 16-bit formats. Thanks to precision tuning and vectorization, execution time is decreased by $12 \%$ and memory accesses are reduced by $27 \%$ on average. As a major outcome, energy consumption is reduced up to $30 \%$.

\section{RELATED WORK}

To overcome the limitations of fixed-format FP types, researchers have proposed multiple-precision arithmetic libraries that perform calculations on numbers with arbitrary precision. ARPREC [1] and MPFR [5] are two widely used libraries which provide support to multiple-precision arithmetic. These libraries are mainly used in contexts where a high-dynamic range is required and higher computation time is considered an 
unavoidable side-effect, such as scientific computing. However they are not suitable to perform explorations of less-than32-bit FP types, since they represent exponents using a full machine word. This approach totally prevents to simulate the behavior of FP types with a reduced number of bits, since tuning of dynamic range is not possible. SoftFloat [9] is a library that implements standard IEEE formats, enabling a bit-accurate emulation of the FP operations performed by FP hardware units. Softfloat can be easily extended to support additional formats, including the ones introduced in this work. However program executions are extremely slow, since the library executes all the computations in software. Moreover any modification to a FP format requires to manually modify code in several source files. Overall, the aforementioned solutions require a full refactoring of the source code; in some cases additional software layers have been introduced to perform this task (e.g., the Boost interval arithmetic library [3]).

Many research tools are available to perform automatic or semi-automatic precision tuning of program variables. In this paper we use DistributedSearch, a tool provided by the fpPrecisionTuning [10] toolchain that finds a near-optimal solution. Its main configuration parameter is the precision of the result, expressed as a value of signal-to-quantization-noise ratio (SQNR) that program outputs must satisfy. This tool requires a binary version of the target program, a target output (i.e., a sequence of FP numbers that are the exact result) and a configuration file. The configuration file should include a list of numbers, which correspond to the precision bits used for program variables. DistributedSearch requires that the target executable is able to read the configuration file to tune the precision of its variables accordingly. In addition, the program must provide its output results on the standard output. On this premises, the tool runs the program multiple times, performing a heuristic search of the minimum precision that can be associated to each variable (for a fixed input set). A second phase performs a statistical refinement to join the precision bindings derived from different input sets. Other tools adopt more advanced techniques but their search space is restricted to standard FP types (e.g., PROMISE [8] and Precimonious [14]), or in other cases they are limited to the analysis of functional expressions (e.g., FPTuner [4] and PRECISA [13]). As a final consideration, all these tool do not enable the analysis of the dynamic range associated to a fixed-format FP type.

On the hardware side, several recent works proposed the design of energy-efficient FP units. Kaul et al. [11] implement a variable-precision multiply-and-add FP unit supporting vectorization. Its configurations use an 8-bit exponent field, while each operand carries a 5-bit certainty field which is processed in parallel with the exponent logic, indicating the number of bits for the mantissa. The certainty field is used to implement automatic precision tracking, which raises the level of precision where it does not meet specified requirements. Considering an energy consumption of $19.4 \mathrm{pJ} / \mathrm{FLOP}$, this solution seems to perform as good as our hardware design. However the memory overhead due to precision tracking and fixed-size exponents is relevant, since the memory transfers are a major contributor to the total energy consumption. Moreover, applications that require 32-bit variables are very inefficient due to repeated operations at lower precision that are performed until a final retry at single precision is executed. Tong et al. [16] explore an iterative (digit-serial) multiplier that can be used inside a FP multiplier. Their design processes 8 bits per cycle, thus operands with up to 8-bits use one cycle, operands with up to 16-bit use 2 cycles, and finally operands up to 24 bits use 3 cycles. Power is reduced by $66 \%$ when using the one-cycle configuration, and by $30 \%$ when using the two-cycles one. Again, single-precision operations become slower and memory effects are not considered. Rzayev et al. [15] explore various smaller-than-32-bit formats for deep learning applications. They introduce a 8-bit FP format that is identical to binary8, showing that vectorization enables higher performance and reduces memory energy used per operation. However they do not propose a mixed-precision FP type system for transprecision computing. Gautschi et al. [6] propose a shared FP unit adopting the logarithmic number system (LNU), which is up to $4.1 \times$ more energy efficient than standard FP unit in non-linear processing kernels. However, LNU is a domain specific approach, and not all FP operations can be implemented.

\section{FLOATING-POINT TYPES AND PROGRAMMING FLOW}

\section{A. Exploration of floating-point formats}

Applications that operate on real-valued data most commonly use IEEE 754-compliant FP formats [18]. Of the standard formats, binary 32 and binary 64 enjoy the most wide-spread use and are also available on general-purpose consumer platforms. While even larger formats are commonly used for scientific computations, reducing the amount of data to process and hence the width of FP formats is more suitable for powerconstrained and embedded platforms. While smaller-than-32bit FP formats (also called minifloats) have been use in computer graphics applications [17], their relevance is rising with the spread of energy-constrained computing platforms, such as near-sensor processing nodes and Internet-of-things endpoints. IEEE formats are packed as the sign bit, $e$ bits for the exponent and $m$ bits for the significand (or mantissa). By choosing a specific format, programmers enforce a trade-off between dynamic range and precision. The dynamic range is the ratio between the largest and smallest representable values, and it is conditioned by $e$. Conversely, the precision is the number of digits of the represented number that are preserved in FP representation, and it is uniquely defined by $m$.

As discussed in Section II, available tools are not flexible enough to simulate arbitrary FP formats by tuning both precision and dynamic range. To enable exploration of arbitrary FP types, we designed a dedicated $\mathrm{C}++$ library, called FlexFloat. This library provides a generic FP type by defining a template class ( $f l e x f l o a t<e, m>$ ) and a set of auxiliary functions for debugging and collecting statistics. Using FlexFloat, all FP types used in the source files can be safely replaced with instantiations of this template class without changing any other part of the program, since class methods include operator overloading. The template parameters include the number of bits used for the exponent $(e)$ and the number of bits used for the mantissa $(m)$, which must be both specified as positive integer values. For instance, flexfloat $<7,12>$ is a FP type including the sign bit, 7 bits in the exponent field and 13 bits in the mantissa field. The FlexFloat library also supports the encoding of denormal numbers, infinities and not-a-number $(\mathrm{NaN})$ values. Arithmetic operations are performed converting the number representation to a native type (e.g., double) and then sanitizing the result, that is adjusting exponent and mantissa to obtain the exact binary representation of the original type. This methodology guarantees shorter execution times w.r.t. emulation approaches (e.g., SoftFloat), and it also produces the same results of a dedicated hardware unit (i.e., precise at bit level). An automatic cast between different template instances is not allowed, so standard arithmetic operations must involve variables of the same instance. This design choice enables a fine-grain control on the intermediate results, since the compiler notifies an error for each operator involving a type mismatch. Consequently, programmers can choose to match the variable types to the same template instance or to insert an explicit cast. A constructor supporting explicit conversions is provided, and it can be used to cast a FlexFloat variable to a different template instance (e.g., 


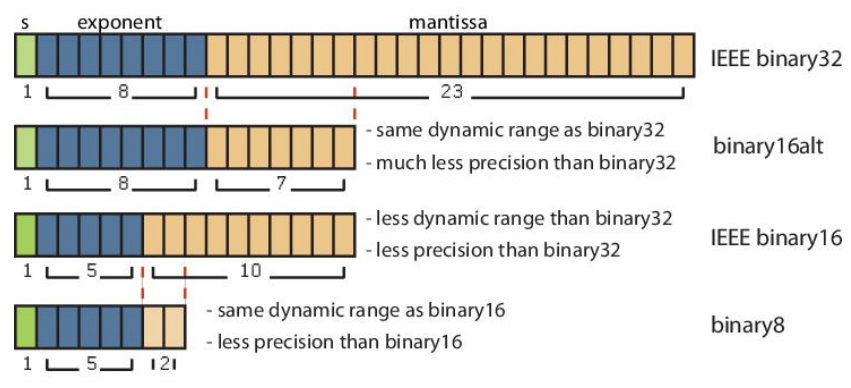

Fig. 1. Overview of floating-point formats used throughout this work.

from flexfloat<el, m1> to flexfloat<e2, m2> ). Constructors with implicit semantics are provided for standard FP types (float, double and long double) to simplify the usage of FP literal values. Vice versa, an automatic cast from a FlexFloat template instance to a standard FP type is not allowed, but it can be performed by invoking an explicit cast operator. This feature can be used to interface sections of source code that use FlexFloat and sections that are strictly bound to standard types (e.g., a call to an external library function whose source code is not available). The main benefits of FlexFloat are:

- it produces binaries that are fast to execute, since its computations rely on native types;

- it reduces the debugging effort, as the compiler performs early check upon template instantiation;

- it is quite intuitive to use, since it provides the usual infix notation for arithmetic operations;

- it can be easily integrated with external tools, having no specific requirements w.r.t. the original source code.

resolved at compile time). To simplify the interaction between a FlexFloat-based program and any external tool, we designed a FlexFloat wrapper, that is a support tool performing three steps: (i) it reads a file specifying a required precision for each program variable, then (ii) it extracts the dynamic range from a configuration file providing the map indexed by precision intervals, and finally (iii) it compiles the program sources providing the derived values for precision and dynamic range as actual parameters in the template instantiations.

To perform an exploration of FP types, we used the DistributedSearch tool introduced in Section II. Since this tool performs precision tuning without considering the dynamic range of variables, we assumed a limited set of initial hypotheses to fix the dynamic range associated to specific intervals of precision bits. Considering our target on ULP systems, we restricted our investigation to 8-bit and 16-bit formats. Among potential 8bit formats, we chose the mapping $(0,3] \mapsto 5$, calling this type binary8. This means that any variable associated to a precision between 1 and 5 bits will be provided with an exponent of 5 bits. This format was conceived to mirror the dynamic range of binary 16 variables. Adopting this convention, conversions between binary 8 and binary 16 only affect precision but do not saturate for values of large magnitudes. Additionally, operations on binary 8 become very cheap in hardware since there are only two explicit mantissa bits. As regards 16bit formats, we considered the mapping corresponding to binary 16 , that is $(0,11] \mapsto 5$, and an alternative mapping that we called binaryl6alt, corresponding to $(0,8] \mapsto 8$. Basically, 8 is the number of bits used for the exponent field in binary32, so this value is a logical upper-bound for any 16-bit format. Again, using the same number of exponent bits of the binary32 format makes conversions much cheaper. Figure 1 summarizes the FP formats used throughout this work.

Table I shows the results of our preliminary analysis, reporting the total number of variables associated to each type. These values are obtained executing DistributedSearch on our

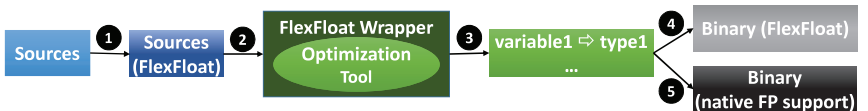

Fig. 2. Overview of the programming flow.

set of benchmarks constrained with a precision of $10^{-1}$. We considered two different configurations of the FP type system, namely V1 (including binary8, binary16, binary32) and V2 (adding binaryl6alt to V1). As a first consideration, binary8 is used for $17 \%$ of the variables in the best case. This format is extremely beneficial in reducing the energy consumption, since it allows to simplify circuitry complexity and it enables vectorization. It is noteworthy that supporting both 16-bit formats contribute in decreasing the number of 32-bit variables in the program w.r.t. the usage of a single 16-bit format. A drawback of binary 16 is that both dynamic range and precision are diminished when compared to binary32. This leads to saturation when converting values with large dynamic from binary32, disqualifying the 16-bit format from being used for transprecision tuning in these cases. Conversely, binaryl6alt features the same dynamic range as binary32, allowing the whole range of values to be converted - albeit with a much larger granularity. In some cases applications do not exploit the dynamic range provided by binaryl6alt, and at the same time they require a higher precision, so our intuition is that we need both types. A further evaluation is provided in Section V-B.

\section{B. Transprecision programming flow}

Figure 2 depicts the transprecision programming flow that we adopted throughout this work. As a first step, application sources are modified to replace standard FP types with multiple instances of flexfloat $\langle e x, m x\rangle$, where $e x$ and $m x$ are variable-specific parameters. Then a tool for precision tuning is invoked (step 2), and different values for $e x$ and $m x$ are explored using the FlexFloat wrapper. After this tuning, program variables are uniquely mapped to supported FP types (step 3). Using this mapping, FlexFloat can provide statistics on the number of operations and casts performed for each FP type which is instantiated (step 4). Moreover, a version of FlexFloat providing explicit template specialization is provided to replace simulated operations with native ones (step 5). This step requires that the compiler for the target platform supports all the FP types provided by the mapping.

\section{TRANSPRECISION FLOATING POINT UNIT}

To evaluate the potential of the FP formats introduced in Section III, we designed a transprecision FP unit supporting vectorization of reduced-precision operations. The hardware unit is built up from three types of slices, each with a fixed width of 32-bit, 16-bit and 8-bit, respectively. Each slice hosts operations on the FP formats that match the slice width, as well as conversion operations. The supported arithmetic operations are addition, subtraction and multiplication. The conversion operations include casts to and from integers (both signed and unsigned) as well as casts among the FP formats. Moreover, the narrower slices are replicated in order to enable subword parallelism inside the unit. Thus, two 16-bit or four 8bit FP operations can be executed simultaneously. Following the single-instruction-multiple-data (SIMD) paradigm, the proposed unit can run scalar operations when only one slice for a given precision is active, and vectorial operation when all the slices of a given precision are active.

TABLE I

VARIABLES CLASSIFIED BY TYPE TYPE USING V1 AND V2 TYPE SYSTEMS.

\begin{tabular}{|l|l|l|l|l|}
\hline & binary8 & binary16 & binary16alt & binary32 \\
\hline V1 & 10 & 29 & - & 72 \\
\hline V2 & 19 & 10 & 41 & 41 \\
\hline
\end{tabular}




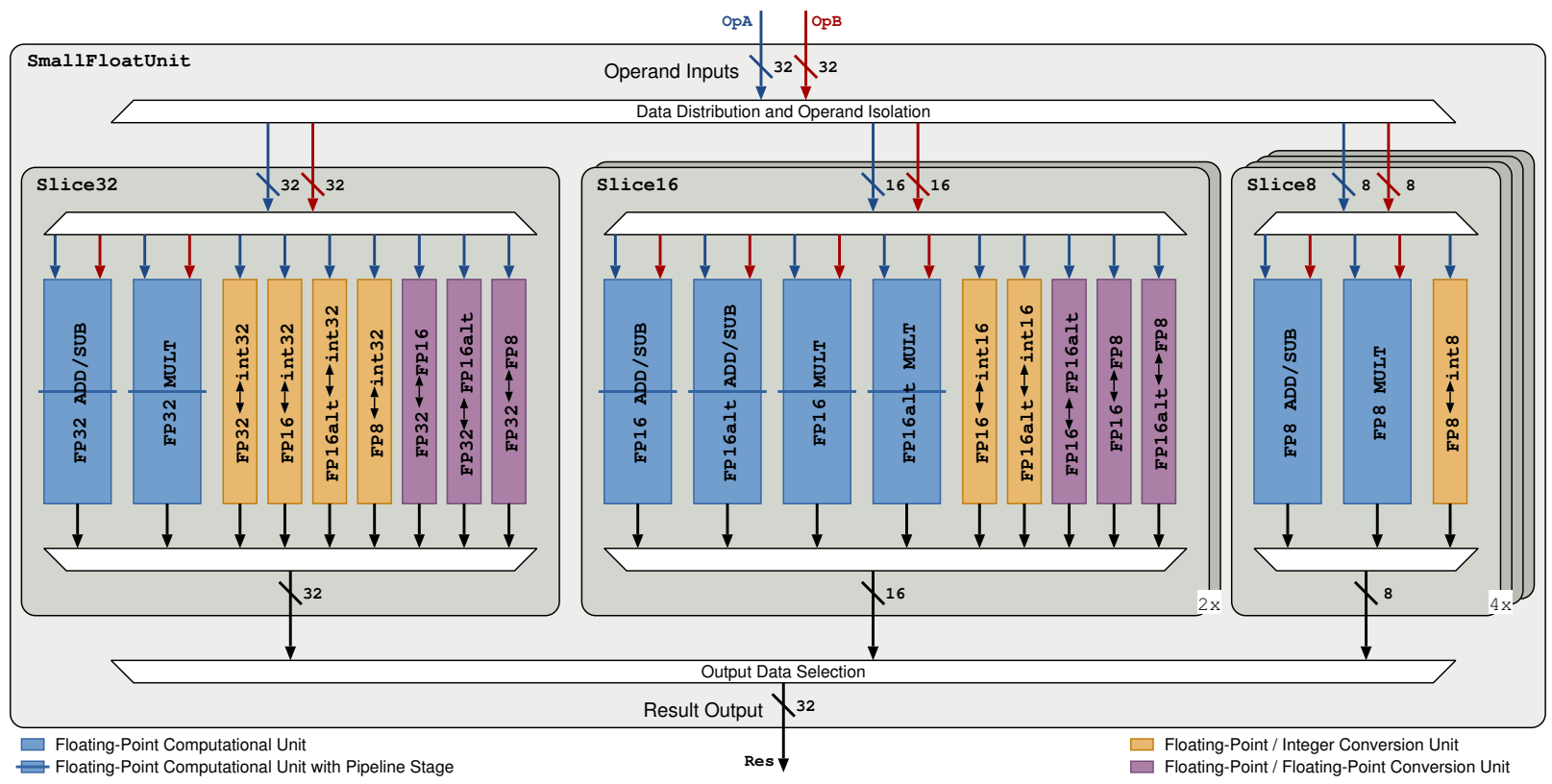

Fig. 3. Simplified block diagram of the designed hardware unit datapath. Control logic as well as data to and from duplicated slices is omitted.

The various individual operation blocks are instances of Synopsys DesignWare FP Datapath components. As a power saving feature, the unit employs operand silencing to all unused operations and formats by forcing zero to prevent transistor switching. To meet the timing requirements of the container core, arithmetic operations in binary32 as well as both 16-bit formats are pipelined with one stage, hence featuring a bandwidth of one operation per cycle and a latency of two clock cycles. Arithmetic operations in binary8 as well as all conversion operations have a one cycle latency. Area optimization of the transprecision FPU and its integration into the core will be completed as future work.

\section{EXPERIMENTAL RESULTS}

\section{A. Evaluation Methodology}

Experiments have been performed on a set of applications which implement key algorithms for two domains of ULP systems, near-sensor computing and embedded machine learning:

- JACOBI applies the Jacobi method to a 2D heat grid:

- KNN computes the k-nearest neighbors of an input value using euclidean distance;

- PCA performs the principal component analysis;

- DWT computes the discrete wavelet transform;

- SVM is the prediction stage of a support vector machine;

- CONV implements a $5 \times 5$ convolution kernel.

Precision tuning has been performed using the fpPrecisionTuning toolsuite on an $\mathrm{x} 86$ workstation, adopting the programming flow described in Section III-B. Since subword vectorization is not supported by the current FlexFloat implementation, program sections that are vectorizable are manually tagged in the source code, and the library provides a distinct report for vectorial operations and casts. The application sources have been compiled using the GCC compiler with a RISC-V backend optimized for PULPino, which provides support for the single-precision FP type defined in the RISC-V instruction set architecture (ISA). Binaries have been executed on the PULPino virtual platform, which is cycle accurate and provides detailed statistics. The virtual platform reports the number of cycles required to execute each instruction that is used in the binary file, targeting the whole program or delimited code regions. The current version of GCC does not include a set of instructions to handle binary16, binaryl6alt and binary 8 formats. Since the latency of binary 16 operations is the same of binary32 ones, we have used the binary 32 type to measure the exact number of cycles required by each instruction to execute. This value depends by the ability of the compiler to schedule other classes of operations (non-FP, binary 8 or casts) to fill latency cycles and avoid stalls in the core pipeline, so it is strictly dependent on both application and compiler back-end. binary8 operations and FP casts always require a single cycle, so their contribution to execution time has been accumulated analytically.

For evaluation of the hardware architecture, the design unit was synthesized for the UMC $65 \mathrm{~nm}$ technology using worst case libraries $\left(1.08 \mathrm{~V}, 125^{\circ} \mathrm{C}\right)$. To have an accurate estimation of the power consumption of the transprecision FPU, we performed post-place-\&-route power simulations. The target frequency for the post-layout design was set to $350 \mathrm{MHz}$, using worst-case conditions. Results take into account the switching activity of input and output registers, added at the boundaries of the unit to evaluate their performance, negligible with respect to the power of the arithmetic units themselves. Energy costs of FP operations were obtained through simulation of the post-layout design in all modes of operation, again using worst-case conditions. Values provided to the unit were generated in a random fashion, making sure that no invalid values were generated. Namely, no NaN or infinity values were applied and operands were chosen sufficiently close to each other such that operand cancellation would not occur during addition or subtraction. For conversions, only values that can be mapped to the target type were applied to eliminate over- or underflow. This was done to simulate normal operation wherein operation is done on meaningful data, while operand cancellation or invalid inputs lead to significantly diminished switching activity inside the operational units. The energy cost of each non-FP instruction executed by the core includes contribution of core logic, instruction memory and data memory. Even if the transprecision FPU has not been integrated in the PULPino core yet, to collect energy measures we have considered the contribution of moving data to/from input and output registers of the FP unit, and also the cost of idle cycles due to pipeline stalls (for both 16-bit and 32-bit instructions). 


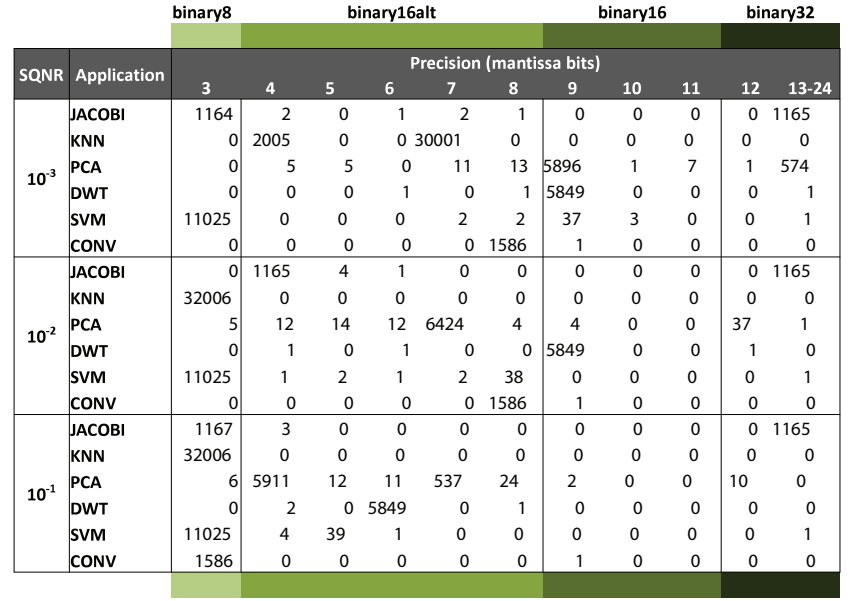

Fig. 4. Precision tuning of program variables for three precision requirements.

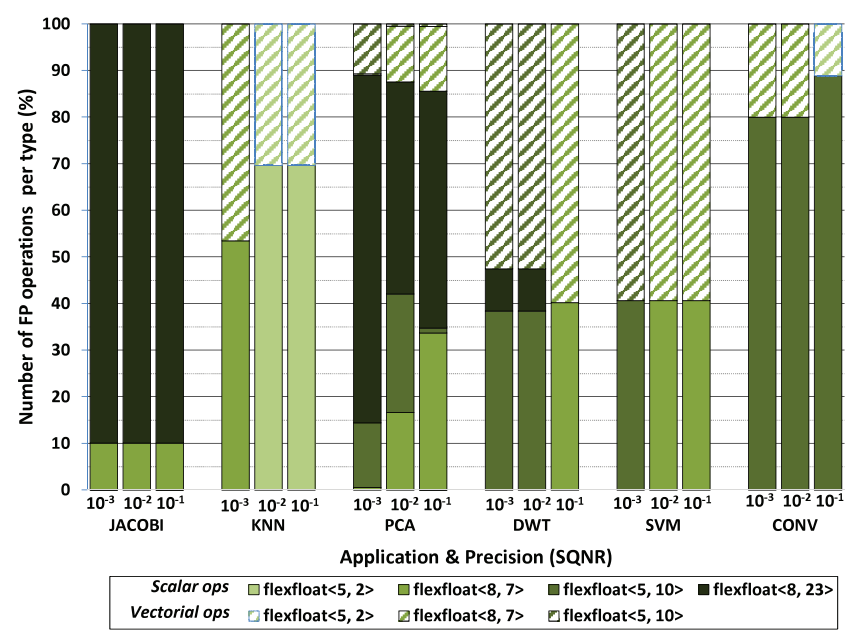

Fig. 5. Breakdown of FP operations for three precision requirements.

\section{B. Precision Tuning}

The table in Figure 4 shows the results of the precision tuning, performed for three precision requirements (SQNR $=10^{-3}, 10^{-2}, 10^{-1}$ ). Rows correspond to applications and columns to precision bits. The reported values represent the number of memory locations (scalar variables or array elements) requiring the minimum number of bits of their column to meet precision constraints. Color bands show the mapping between precision bits and the FP type system introduced in Section III. KNN and SVM make wide use of binary8 data, while in general other application do not. In fact binary8 emerges a format which is profitable in specific applications domains, while binary 16 is a good candidate for a wider use. Moreover, it is evident that most elements in the interval $[9,11]$ are concentrated in column 9 , which is the minimum number of precision bits required for a binaryl6 type. This is due to the fact that these elements strictly require the additional precision provided by binary 16 w.r.t. binaryl6alt, which means that both types are useful in different contexts. For the same reason, variables in column 4 are more than variables in column 5 , since they include all cases that do not require a wider dynamic range w.r.t. binary8 (regardless of the precision). Conversely, variables that require high precision usually require more than 12 precision bits, and they are concentrated in the last column.

\section{Execution time and memory accesses}

Figure 5 shows a breakdown of the FP operations performed by each application, taking into account the same precision

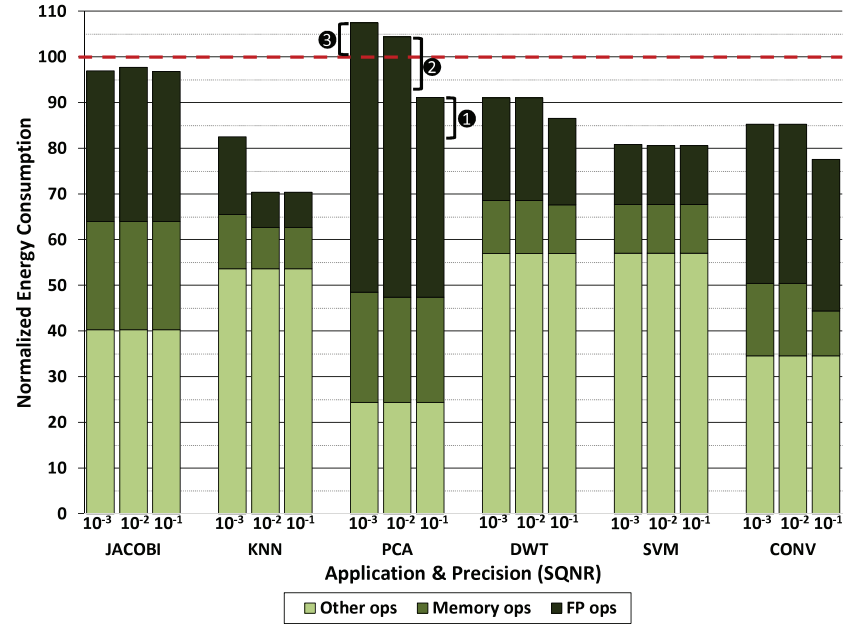

Fig. 7. Energy consumption normalized to binary 32 baseline.

requirements of previous section. This is a dynamic view of the FP type system at run-time (whereas Figure 4 provides a static view after precision tuning). Each bar segment quantifies the contribution of a specific type to the total number of FP operations, discriminating scalar and vectorial operations. In JACOBI and PCA there is a major contribution of 32bit operations, which is a first trait that adversely affects a potential reduction of energy consumption. Another negative aspect is the lack of vectorial operations, that is pathological in JACOBI. In this work we have not considered any advanced coding techniques (e.g., manual code vectorization), but we have based out analysis on off-the-shelf versions of applications that could be further optimized to achieve following the guidelines derived from our considerations.

Figure 6 depicts two groups of bars for each application, reporting memory accesses and executions cycles. Values are normalized to the binary32 version of the application, that acts as a baseline. Vectorial memory accesses, cycles spent in vectorial operations and cycle spent in cast operations are highlighted with a different pattern. As showed in the previous section, JACOBI does not perform any vectorial operation. Moreover the number of cycles is equivalent to the original version, since this application only uses a limited number of binaryl6alt variables without exploiting vectorization. In the most general case, the number of cycles can even exceed the baseline, since cast operations between different FP types are introduced (e.g., JACOBI when SQNR $=10^{-3}$ ). As a major limitation, current tools for precision tuning do not take into account the cost of casts, since they aim at minimizing the number of precision bits used by any variable and no additional optimization goal can be specified. This effect is further exacerbated in PCA, where the number of casts required after the tuning process exceeds $10 \%\left(\mathrm{SQNR}=10^{-1}\right.$ ) and $20 \%\left(\mathrm{SQNR}=10^{-2}\right.$ and $\left.\left.10^{-3}\right)\right)$. In other benchmarks we can observe evident benefits in memory accesses and cycles, mainly due to vectorization, while the overhead of cast operations is not relevant. SVM shows the maximum reduction of memory accesses, that is $48 \%$, since $60 \%$ of FP operations are vectorizable (for any precision requirement). On average, the execution time is decreased by $12 \%$ and memory accesses are reduced by $27 \%$. Considering JACOBI and PCA as outliers, these values turn into $17 \%$ and $36 \%$.

\section{Energy consumption}

Figure 7 shows the energy consumption of each application, normalized to the binary32 baseline. Each bar contains three contributions, the FP operations (FP ops), the memory accesses (Memory ops) and all the other instructions that are 


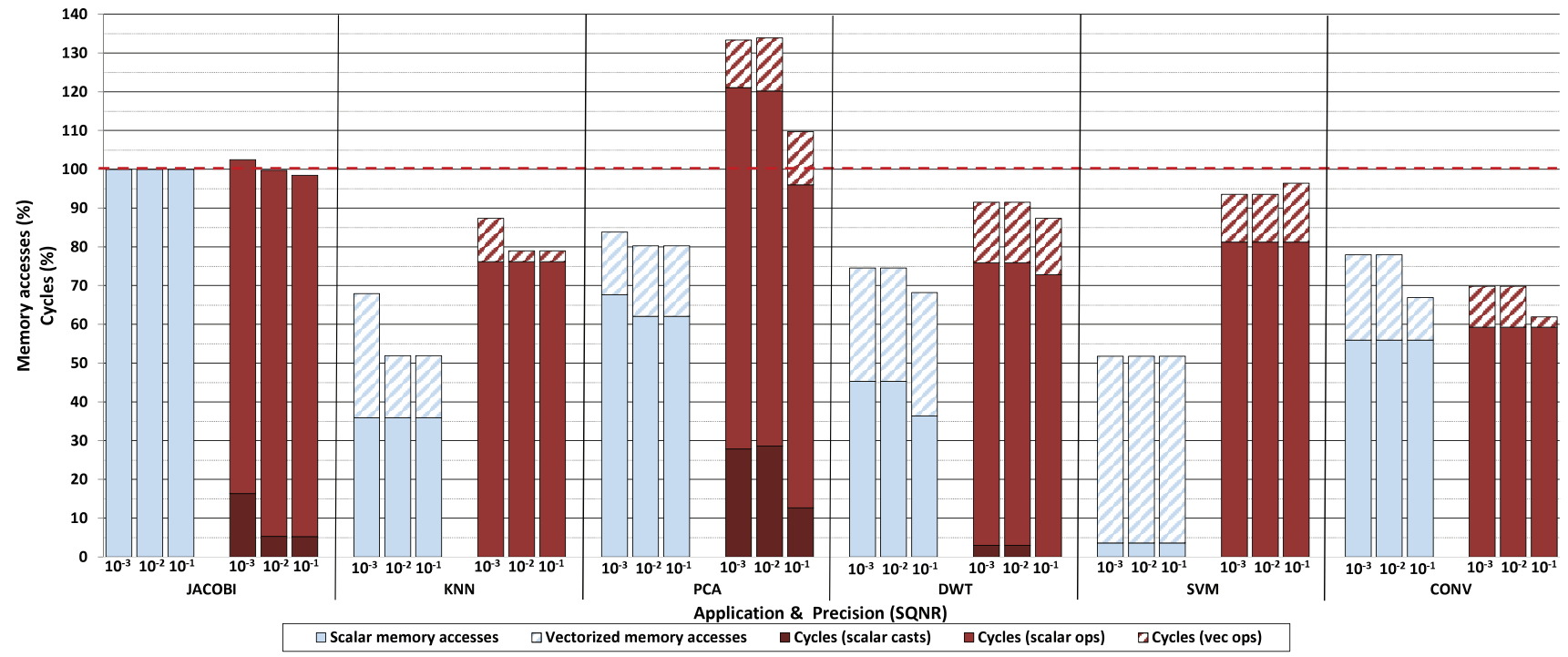

Fig. 6. Memory access and cycles for three precision requirements, normalized to binary32 baseline.

executed by the core (Other ops). These numbers can be easily justified by the considerations in the previous section. On average, the energy consumption of JACOBI is $97 \%$, since this application makes limited use of smaller-than-32bit types and does not exploit the benefits of vectorization. The energy consumption of PCA is $7 \%$ and $8 \%$ greater than the baseline for two precision requirements $\left(\mathrm{SQNR}=10^{-3}\right.$ and $10^{-2}$ ), due to the high number of casts coupled with a predominant number of scalar operations on binary32 values. The other applications have average energy savings around $18 \%$ compared to the baseline, with a maximum of $30 \%$ measured for KNN. Considering the results of Section V-B, the behavior of $\mathrm{KNN}$ is related to three main characteristics, (i) it uses the binary 8 type for all program variables, (ii) it exploits vectorization, and finally (iii) it requires a limited number of non-vectorized memory accesses.

Advanced vectorization techniques can provide huge benefits whenever an application provides a relevant percentage of smaller-than-32-bit operations after the tuning process. To demonstrate this assumption, we applied manual vectorization to PCA, thus reducing the energy consumption to lower values $(101 \%, 96 \%$ and $85 \%)$. These gains are marked on Figure 7 by labels 1, 2 and 3. Further energy savings can be only achieved by reducing the contribution of casts with the support of smarter tools for precision tuning.

\section{CONCLUSION}

This work introduces an extended FP type system with complete hardware support to enable transprecision computing on ULP embedded platforms. Experimental results show that our approach is effective in reducing energy consumption by leveraging the knobs provided by the extended FP type system and thanks to vectorization support. The energy consumption is reduced on average by $18 \%$, and up to $30 \%$ for specific applications. At the same time, execution time is decreased by $12 \%$ and memory accesses are reduced by $27 \%$.

Our future work will be focused on three main aspects. First, the study of new techniques of precision tuning, that take into account the costs of casts with the aim to formulate a multiobjective optimization problem. Second, the optimization of transprecision hardware units, to achieve better performance and minimize the area. Third, the investigation of compiler passes and vectorization techniques to better exploit transprecision opportunities at compile time.

\section{ACKNOWLEDGMENT}

This work has been partially supported by the European FP7 ERC project MULTITHERMAN (g.a. 291125) and by the European H2020 FET project OPRECOMP (g.a. 732631).

\section{REFERENCES}

[1] D. H. Bailey et al., "ARPREC: An arbitrary precision computation package," Lawrence Berkeley National Laboratory, 2002.

[2] C. Bekas et al., "Low-cost data uncertainty quantification," Concurrency and Computation: Pract. \& Exper., vol. 24, no. 8, pp. 908-920, 2012.

[3] H. Brönnimann et al., "The design of the Boost interval arithmetic library," Theoretical Comp. Science, vol. 351, no. 1, pp. 111-118, 2006.

[4] W.-F. Chiang et al., "Rigorous floating-point mixed-precision tuning," in Proc. of the 44th ACM SIGPLAN Symposium on Principles of Programming Languages. ACM, 2017, pp. 300-315.

[5] L. Fousse et al., "MPFR: A multiple-precision binary floating-point library with correct rounding," ACM Transactions on Mathematical Software (TOMS), vol. 33, no. 2, p. 13, 2007.

[6] M. Gautschi et al., "An Extended Shared Logarithmic Unit for Nonlinear Function Kernel Acceleration in a 65-nm CMOS Multicore Cluster," IEEE Journal of Solid-State Circuits, vol. 52, no. 1, pp. 98-112, 2017.

[7] - "Near-Threshold RISC-V Core With DSP Extensions for Scalable IoT Endpoint Devices," IEEE Transactions on Very Large Scale Integration (VLSI) Systems, 2017.

[8] S. Graillat et al., "Auto-tuning for floating-point precision with Discrete Stochastic Arithmetic," 2016.

[9] J. R. Hauser, "Handling floating-point exceptions in numeric programs," ACM Transactions on Programming Languages and Systems (TOPLAS), vol. 18 , no. 2 , pp. 139-174, 1996.

[10] N.-M. Ho et al., "Efficient floating point precision tuning for approximate computing," in 22nd Asia and South Pacific Design Automation Conf. (ASP-DAC). IEEE, 2017, pp. 63-68.

[11] H. Kaul et al., "A 1.45GHz 52-to-162GFLOPS/W variable-precision floating-point fused multiply-add unit with certainty tracking in $32 \mathrm{~nm}$ CMOS," in IEEE Int. Solid-State Circuits Conf., 2012, pp. 182-184.

[12] P. Klavík et al., "Changing computing paradigms towards power efficiency," Phil. Trans. R. Soc. A, vol. 372, no. 2018, 2014

[13] M. Moscato et al., "Automatic Estimation of Verified Floating-Point Round-Off Errors via Static Analysis," in Int. Conf. on Computer Safety, Reliability, and Security. Springer, 2017.

[14] C. Rubio-González et al., "Precimonious: Tuning assistant for floatingpoint precision," in Proc. of the Int. Conf. on High Perf. Computing, Networking, Storage and Analysis. ACM, 2013, p. 27.

[15] T. Rzayev et al., "DeepRecon: Dynamically reconfigurable architecture for accelerating deep neural networks," in Int. Joint Conf. on Neural Networks (IJCNN), 2017, pp. 116-124.

[16] J. Y. F. Tong et al., "Reducing power by optimizing the necessary precision/range of floating-point arithmetic," IEEE Transactions on Very Large Scale Integration (VLSI) Systems, vol. 8, no. 3, pp. 273-286, 2000.

[17] M. M. Trompouki and L. Kosmidis, "Towards general purpose computations on low-end mobile GPUs," in Design, Automation \& Test in Europe Conf. \& Exhibition (DATE). IEEE, 2016, pp. 539-542.

[18] D. Zuras et al., "IEEE standard for floating-point arithmetic," IEEE Std 754-2008, pp. 1-70, 2008. 